\title{
Memory strength and recency judgments
}

\author{
DOUGLAS L. HINTZMAN \\ University of Oregon, Eugene, Oregon
}

\begin{abstract}
Two experiments were done to examine the effect of memory strength on numerical judgment of recency (JOR). In one experiment, the strong versus weak manipulation was defined by stimulus type (pictures vs. names); and in the other, it was defined by long versus short study durations of pictures. Two hypotheses were contrasted: (1) that strong items seem more recent than weak items (the activation hypothesis), and (2) that JORs are more accurate for strong items than for weak items (the accuracy hypothesis). Results of both experiments supported the activation hypothesis. On the basis of these and previous results, it is argued that recency is inferred partly from a memory's strength or activation and partly from some other time-related clue to the age of the memory.
\end{abstract}

The philosopher David Hartley gave an intuitively plausible explanation of how one infers the recency of a remembered event: "If the specific nature of memory consists in the great vigour of ideas ... then, as this vigour abates, it ought to suggest to us a length of time elapsed; and vice versa ..." (Hartley, 1834, p. 137). To paraphrase Hartley in more modern terms, one might say that an optimal cue activates strong traces more than weak traces, that memory strength declines with time, and that apparent recency is inferred from trace activation. In the present article, this idea will be referred to as the activation hypothesis. Taken by itself, the hypothesis predicts that if two memories differ in strength, the stronger one will seem the more recent. A mathematical model of recency judgment by Hinrichs (1970) represents one instantiation of the activation hypothesis.

But the concept of memory strength implies more than what Hartley called "vigour." Strong memories are usually thought to be more accurate than weak memories. If that accuracy extends to information about the memory's age, then a judgment of recency (JOR) based on a strong memory should be more accurate than a JOR based on a weak memory. This idea will be referred to here as the $a c$ curacy hypothesis. To make the accuracy hypothesis more explicit, it may be related to an oscillator-based model of memory for time, proposed by G. D. A. Brown, Preece, and Hulme (2000). According to this model, when an event is encoded in memory, it is associated with the states of several oscillators with varying frequencies. When the event is remembered, the retrieved oscillator states are compared with the current oscillator states, and the difference is turned into a JOR. If we make the reasonable assumption that strong memories are better associated with their oscillator states than weak memories are, we may

Correspondence should be addressed to D. L. Hintzman, Department of Psychology, University of Oregon, Eugene, OR 97403 (e-mail: hintzman@ uoregon.edu). predict that JORs for strong items will be more accurate than those for weak items.

In a typical numerical JOR experiment, subjects go through a long, uniform list of items that lacks temporal landmarks and associative structure. Individual items are repeated at a variety of lags, where the lag is defined as the number of items intervening between the study and test trials. On test trials, subjects make JORs (i.e., lag judgments) from a range of values specified by the experimenter. If this range corresponds closely to the range of actual lags, mean JOR follows a predictable pattern in which it overshoots the correct values at short lags and undershoots the correct values at long lags, because errors tend to "regress" toward the middle of the JOR scale (Hinrichs, 1970). Because the accuracy hypothesis predicts more such regression for weak items than for strong items, the result should be a crossover interaction. The two hypotheses thus make different predictions regarding the effects of strength on JOR, particularly at the longest lags (see Figure 1). What is crucial here is the crossover pattern, which is necessary for strong-item JOR to be more accurate at both long lags and short lags.

To examine this question, we need to distinguish it from two other issues: First, one should not confound the effect of strength or activation on JOR with its effect on recognition memory, because subjects may use different standards to assign JORs to items they fail to recognize. This problem can be minimized by requesting JORs only for items that are recognized as old. Of course, conditionalizing on recognition may reduce the difference between strong and weak items, but it should not eliminate the difference. Second, as was done by Hinrichs (1970), one needs to distinguish between the concept of trace strength-a hypothetical property of the memory of an individual event-and the concept of cumulative strength-a hypothetical effect of repetition. An item that occurred twice previously has two recencies, and memory-judgment studies suggest that this is true not only objectively, but also subjectively (Flexser \& Bower, 1974; Hintzman, 2003a). Thus, the present 

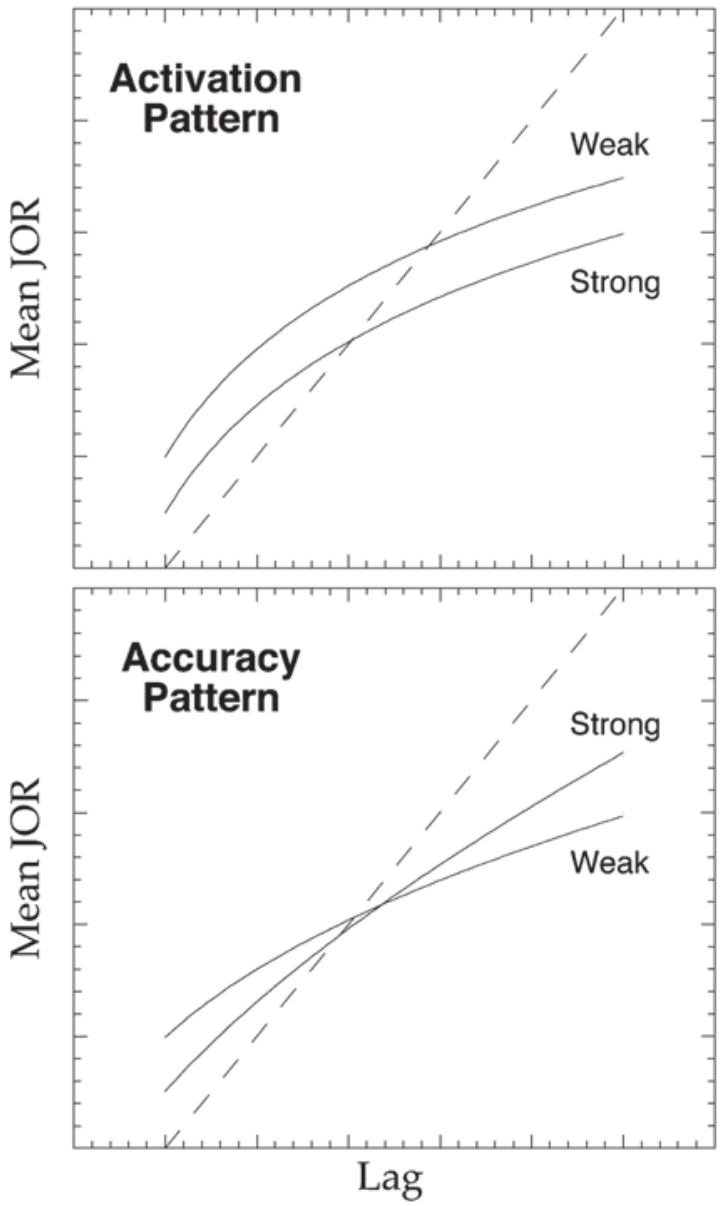

Figure 1. Patterns of mean JOR predicted by the activation hypothesis and the accuracy hypothesis. Diagonal lines indicate perfect accuracy.

article is concerned with trace strength and will not deal with cumulative strength or multiple repetition.

In an early study that met these conditions, Fozard and Weinert (1972) compared JOR for pictures versus words. There were three lag conditions, which averaged 13.5, 25.5, and 49.5 intervening items. In keeping with the literature on the mirror effect (e.g., Glanzer \& Adams, 1985), recognition memory was better for pictures than for words. Median JORs, conditional on correct recognition, were lower for pictures than for words at the shortest lag, but they were about the same as those for words in the two longer lag conditions. In literature reviews, this result has been taken as evidence for the accuracy hypothesis (e.g., see Friedman, 1993; Hintzman, 2000). However, a more cautious interpretation is that the result is ambiguous, because it fits the activation pattern as well as it does the accuracy pattern (see Figure 1). Fozard and Weinert did not counterbalance materials over lags, and they computed medians rather than means, possibly because their JOR scale lacked an upper limit. The design may have been insufficient to yield a definitive outcome.
Two other relevant experiments compared memory judgments for different categories of words (Hintzman, 2003b). Rare words were recognized better than common words, and concrete words were recognized better than abstract words-both results suggesting a difference in strength, consistent with the mirror effect. In both experiments, mean conditional JOR was shorter for the better recognized class of words at every lag, ranging from 5 to 30 items - a result consistent with the activation pattern.

A different manipulation suggests that the crucial variable is not the strength of the memory trace, per se, but the degree to which the trace is activated by the retrieval cue (Hintzman, 2002). In this study, the stimuli were first names presented in two different visual contexts or fonts. Again, the lags and JORs ranged from 5 to 30 items. When a name's study and test contexts were the same, recognition was better than when the two contexts were different, and mean conditional JOR was also shorter. This result was interpreted as consistent with either of two hypotheses concerning the basis of JOR. One was the activation hypothesis, which subsumes the assumption that a cue that matches the trace produces greater activation. The other was the context change hypothesis, which holds that the basis of JOR is a comparison of the retrieved context and the test context-the better the two contexts match, the shorter should be the JOR.

In a more recent study, however, I rejected the context change explanation of JOR (Hintzman, 2004). It was reasoned that cognitive context should change more rapidly, the more items are processed per unit of time. The effects of time and items on JOR can be dissociated by varying the rate of presentation. In two experiments that accomplished this, JOR proved to be based on the memory's age, as measured in time, and independent of the number of items processed during the interval. By a process of elimination, this conclusion suggests that the physical matching result reported in Hintzman (2002) was mediated by differential activation.

With one exception (Fozard \& Weinert, 1972), which yielded an ambiguous outcome, the studies just reviewed appear to support the activation hypothesis over the accuracy hypothesis, but there are two reasons to be cautious about this conclusion. First, the expectation that judgments based on strong memories should be more accurate than judgments based on weak memories seems intuitively compelling. Second, the extant results raise questions about the generality of the activation pattern, over both materials and lags. The present experiments were intended to explore these issues further. In Experiment 1 , JORs were compared for color photographs versus names - an item manipulation similar to that of Fozard and Weinert, which was expected to yield a large strength difference. In Experiment 2, all stimuli were of a single type (color photographs), and memory strength was manipulated through study duration. In both experiments, the lags ranged from 9 to 60 , and the possible JORs ranged from 10 to 60 , doubling the range of lags used in the Hintzman $(2002$, 2003b) experiments. 


\section{EXPERIMENT 1}

\section{Method}

Subjects. Fifteen University of Oregon students were tested individually in sessions lasting less than $50 \mathrm{~min}$. Eight participated for pay, and 7 participated for course credit.

Materials. There were two types of visual stimuli: names and pictures. The former were common female and male first names, taken from a 1990 U.S. Census Web site. The initial name list was edited to remove close variants and names that were visually similar, yielding a final list of 400 names, three to eight letters in length. Each name was displayed on the computer screen in black uppercase 32-point Helvetica font, against a white background. The pictures were 216 digitized color photographs, mostly from a Microsoft Office CD-ROM clip-art collection. They were selected to be of low interitem similarity and depicted a wide variety of scenes and objects. A typical picture occupied a screen area of about $230 \mathrm{~cm}^{2}$.

Procedure. Each subject was seated before a computer controlling a 15-in. monitor. The subjects were told that they would see a long list of names and pictures, many of which would be repeated. They were informed that each stimulus would remain on the screen until they pressed one of seven keys of the keyboard. If the item was new, they were to press the " $Z$ " key, which had been labeled with an "N." If they thought the item was old, they were to give a JOR - that is, a judgment of how many items back in the list it had been when they saw it before. JORs were made using the 1-6 keys of the numerical keypad, by multiplying by 10 ; thus, the possible values for JOR were 10, 20, 30, 40, 50, and 60 items.

The presentation list was 600 items long. Items were selected from the name and picture master lists randomly, without replacement. The experimental program stepped through positions $\mathrm{P}=$ $1-600$, in sequence. It first checked to see whether a repetition had already been assigned to $P$. If not, it selected either a name or a picture, with equal probability, and assigned it to P. Next, the program randomly chose one of four repetition lags (lag $=9,21,38$, or 60 ) and checked to determine whether position $\mathrm{P}+$ lag was already occupied. If that position was empty, the item in $\mathrm{P}$ was assigned also to P + lag; otherwise the item was not repeated.

Excluding the first 62 trials, which were not analyzed, this design yielded an average of 29 observations per cell, per subject. Each item remained on the screen until the subject responded. A 500-msec blank interval preceded each trial. The subjects tended to go through the list at a regular pace, typically completing the task in 25-35 min.

\section{Results}

Data from 2 outlying subjects were dropped from further analysis. One had a recognition hit rate of less than $70 \%$, and the other had mean JORs that were essentially uncorrelated with lag $(r<.1)$. Statistical tests for the remaining 13 subjects were based on $t$ tests for planned contrasts-dichotomous for item type and linear for lag (see Rosenthal \& Rosnow, 1985).

Recognition. Overall recognition performance was measured using $\operatorname{Pr}=P($ hit $)-P$ (false alarm) . As expected, recognition was better for pictures than for names $[\mathrm{Pr}=$ .958 vs. $.835 ; t(12)=4.950, p<.001]$. False alarms and hits both showed the mirror effect pattern. Mean false alarm rates on pictures and names were .015 and .113 , respectively $[t(12)=3.363, p<.001]$, and mean hit rates were .973 and $.948[t(12)=2.579, p<.05]$. As is shown in the top panel of Figure 2, hit rates also displayed a significant linear trend on lag $[t(12)=5.479, p<.005]$, but the lag $\times$ item type interaction was not reliable $(t<1)$.

JOR. Mean JOR is shown in the bottom panel of Figure 2 . In addition to the obvious effect of lag, JOR dis-
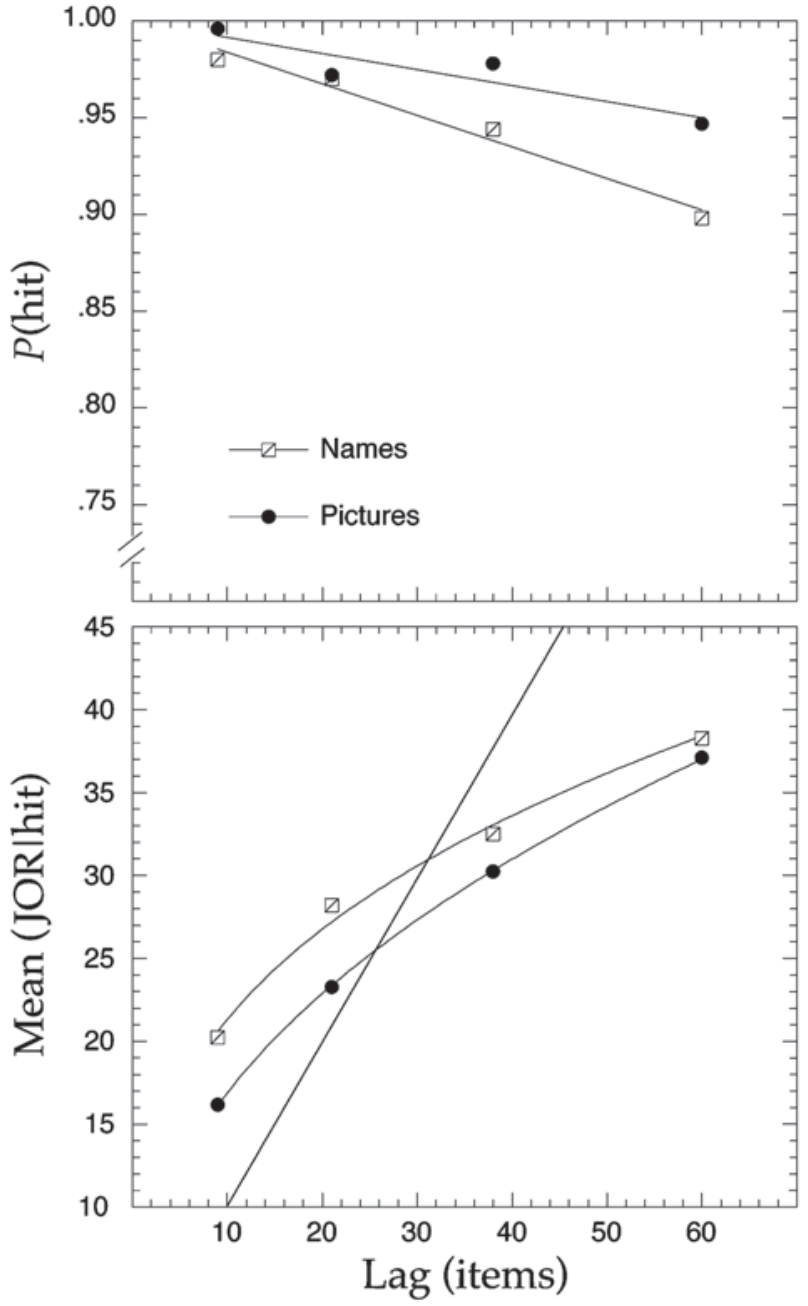

Figure 2. Results of Experiment 1, as a function of lag and item type. Top panel: Probability of a recognition hit $(S E=.01)$. Bottom panel: Mean JOR conditional on a hit $(S E=0.8)$. Both $S E S$ were computed from the between-conditions mean square (see Loftus \& Masson, 1994).

played an effect of item type $[t(12)=5.313, p<.001]$ and a lag $\times$ item type interaction $[t(12)=3.325, p<$ .01]. Despite the interaction, pictures were judged to be more recent than names at every lag, and if the analysis was restricted to the two longest lags, the difference remained significant $[t(12)=2.697, p<.02]$. The result is consistent with the activation hypothesis.

Salient differences between names and pictures, however, make the JOR difference theoretically ambiguous. The nature of retrieved temporal information could be different for names and for pictures, or the same temporal information could be mapped onto JOR according to different standards. In the model of Hinrichs (1970), decaying strength is transformed into numerical JOR through a process of Thurstonian scaling, which can be affected by instructions to subjects regarding the range of possible lags. In a similar way, self-instruction could differentially affect the way in which recency information for pictures 
and names is transformed into a numerical JOR. It might be argued that such differential mapping is unlikely when pictures and names are randomly intermixed, as they were in Experiment 1, because it would require subjects to constantly readjust their JOR criteria. Nevertheless, the possibility of differential scaling makes it difficult to unambiguously infer differences in strength from the obtained JOR differences.

\section{EXPERIMENT 2}

The aim of Experiment 2 was to isolate the effect of strength from possible scaling or bias differences, by manipulating strength in a way that is not confounded with type of item. The weak and strong stimuli were color pictures, studied for either a short duration or a long duration.

\section{Method}

Twenty University of Oregon students participated for course credit. The pool of photographs used in Experiment 1 was expanded to include 370 pictures. The experimental design was like that of Experiment 1, except that weak and strong items were defined by a short (1,500-msec) study duration and a long (3,500-msec) study duration. From the subject's viewpoint, every presentation was either a study trial or a test trial. On a study trial, exposure duration was controlled, and the subject was not required to make a keypress. On a test trial, the word TEST was superimposed in the center of the picture in white, 32-point Helvetica font, and the subject had to make one of seven responses_- "N," or a numerical JOR_as had been required on all trials in Experiment 1.

Except for the distinction between study and test trials, the algorithm that generated the list order was the same as that used in Experiment 1. Each picture was displayed in the test-trial format on its final appearance. Thus, if the item was not scheduled to be repeated, Trial 1 was a test, and if the item was repeated, Trial 2 was a test. The subjects were instructed about the two study durations and the difference between study trials and test trials. Otherwise, the procedure was the same as that of Experiment 1.

\section{Results}

The data of 2 subjects who had recognition hit rates below $70 \%$ were dropped from further analysis, leaving an $N$ of 18. Data from the retained subjects are shown in Figure 3.

Recognition. As expected, recognition performance was better on long duration pictures than on short duration pictures $[\mathrm{Pr}=.82$ vs. .76; $t(17)=4.846, p<.001]$. The single false alarm rate, computed from items tested on Trial 1, was .04. The top panel of Figure 3 shows recognition hit rates for items that were tested on Trial 2. In addition to the main effect of duration, hit rates displayed a significant effect of lag $[t(17)=4.760, p<.001]$ and a nonsignificant duration $\times$ lag interaction $[t<1]$.

JOR. The bottom panel of Figure 3 shows mean JOR for items correctly identified as old. There was a reliable main effect of study duration $[t(17)=3.652, p=.002]$. At every lag, long-duration items were judged to be more recent than short-duration items. Although the duration effect was smallest at Lag 60, the lag $\times$ duration interaction was not reliable $(t<1)$.

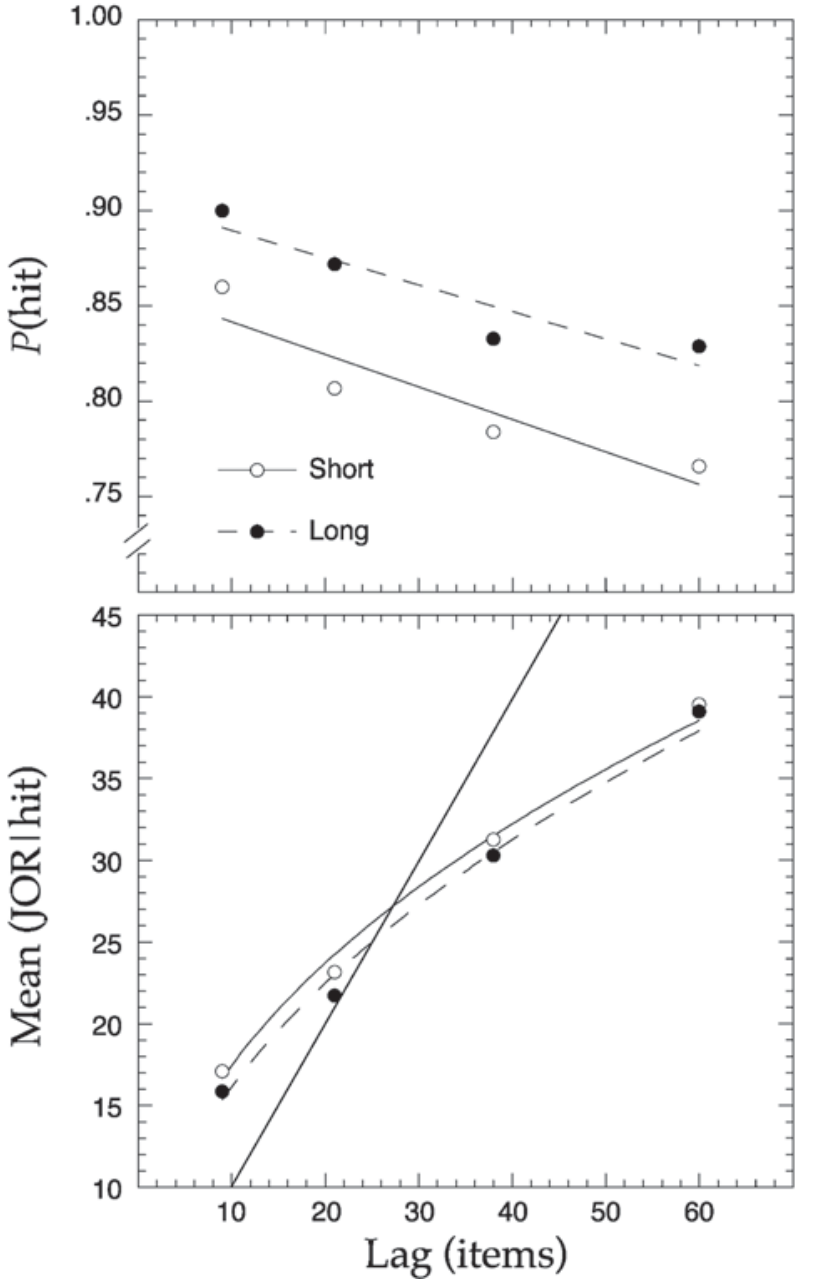

Figure 3. Results of Experiment 2, as a function of lag and item type. Top panel: Probability of a recognition hit $(S E=.02)$. Bottom panel: Mean JOR conditional on a hit $(S E=0.7)$. Both $S E S$ were computed from the between-conditions mean square (see Loftus \& Masson, 1994).

\section{DISCUSSION}

Do stronger memories seem more recent than weaker ones, regardless of actual recency (the activation hypothesis), or is JOR more accurate for stronger memories (the accuracy hypothesis)? The data of Experiment 1 conformed to the activation pattern, in that pictures were judged more recent than names. Although mean JOR displayed a strength $\times$ lag interaction, it was not a crossover interaction of the kind specified by the accuracy hypothesis. This result appears to clarify the ambiguous outcome of Fozard and Weinert (1972), in favor of the activation hypothesis. The alternative explanation of Experiment 1 - that subjects used different standards to map JORs onto strength with pictures and with names-would not apply to Experiment 2, in which exposure duration was used to manipulate the strength of a single type of 
item. The results of this experiment again conformed to the activation pattern: Long-duration pictures were given reliably lower JORs than were short-duration pictures, and there was no crossover interaction.

One might object that the JOR difference in Experiment 2 appears to be small, but — as will be seen later - the magnitude of the effect (about 1 item on average) is about what would be expected from the results of other studies that used the same running memory task. Moreover, it can be argued that the effect of strength was actually somewhat greater than Figure 3 suggests. This is because lag is defined relative to the offset of the study trial, and this requires the onsets of study trials for long-duration pictures to be $2,000 \mathrm{msec}$ earlier than those for shortduration pictures. If a memory's subjective age is tied to its initial encoding, then long-duration memories might be expected to seem slightly older at test than short-duration memories - the opposite of the observed difference in JOR. If this is right, then the duration-induced difference in activation or strength must have been powerful enough to outweigh this time-of-encoding difference-perhaps $30 \%$ larger than it appears in the data.

A final consideration is that the outcome of Experiment 2 is consistent with the outcomes of four other running JOR experiments conducted in the same laboratory under similar conditions. These experiments compared high-frequency and low-frequency words (Hintzman, 2003b, Experiment 1), abstract and concrete words (Hintzman, 2003b, Experiment 2), matching and nonmatching physical context (Hintzman, 2002), and names and pictures (the present Experiment 1). In each case, the outcome resembled the activation pattern shown in Figure 1 - that is, there was a main effect, and no indication of a crossover interaction. In most cases, strong items were more sensitive to recency, in that the JOR curve for strong items was steeper than that for weak items. Nevertheless, strong items were judged less accurately than weak items at the longest lags, contrary to the accuracy hypothesis. The consistent tendency for the curves to converge at longer lags may simply mean that the subjective difference between JORs of 10 and 20 is greater than that between JORs of 50 and 60 (e.g., Schneider, Parker, Ostrosky, Stein, \& Kanow, 1974).

Of course, one cannot completely discount the possibility that the curves would cross if the range of lags were further extended, but this would require two reversals of JOR accuracy. That is, JOR accuracy for strong items would have to be greater than that for weak items at short lags, lower at intermediate lags (i.e., long lags in the present experiments), but then greater again when lags are considerably longer. Moreover, in a study of memory for news items from previous years, well-remembered events were judged to be more recent than more poorly remembered events of equivalent age (N. R. Brown, Rips, $\&$ Shevell, 1985). This suggests that the activation pattern generalizes to lags far beyond those of the present experiments.
In each of the running JOR experiments conducted in my laboratory, a strength or activation difference was found in JOR and also in recognition memory. Is there a simple relation between these two outcomes? One way to explore this question is to graph the strong versus weak difference in JOR against the corresponding difference in recognition accuracy, as measured by Pr. Figure 4 is a scatter diagram showing the results of all five experiments. The correlation $(r=.895)$ suggests that the effects of strength or activation in the two tasks might be attributed to the same underlying process. The biggest procedural difference among the plotted experiments was the range of lags, which was 5-30 items in the earlier studies, as opposed to 9-60 items in the present experiments. There is no suggestion in Figure 4 that the observed relation can be attributed to this procedural difference.

Despite the commonality suggested by Figure 4, there is at least one important difference between JOR and recognition. As one would expect on intuitive grounds, lag has a stronger effect on JOR than on recognition. This difference can be quantified in the results of the present experiments, by contrasting the variance explained by linear trend on lag with the variance explained by the item manipulation. As can be seen in Table 1, when compared with the item manipulation, lag has a disproportionate effect on JOR. Previous studies using the running JOR task all produced similar outcomes. Moreover, Hintzman (2003b) directly compared JOR with recognition confidence ratings, conditionalizing both measures on correct recognition. The effect of lag on JOR was much stronger than its effect on recognition confidence. The activation hypothesis by itself does not explain this difference between JOR and recognition.

Further evidence that some unique cue contributes to JOR comes from the study of fast versus slow presentation rates, mentioned earlier (Hintzman, 2004). When contributions of time and items are dissociated, JOR appears to be based on the passage of time, with no independent contribution from the number of items. This outcome suggests that JOR is especially sensitive to some unknown, time-specific cue, which we might call "factor $T$." Context change apparently can be ruled out as the basis of factor $T$, because cognitive context should change more rapidly in fast blocks than in slow blocks.

Then what is factor $T$ ? One possibility is that JOR is based on retrieved oscillator readings that were associated with the event at encoding (G. D. A. Brown et al., 2000). This could help explain why JOR is related to time rather than items, but it does not explain why, at the longest lags, JOR is less accurate for strong items than for weak items. The failure to obtain a crossover interaction is especially troublesome in Experiment 2, where differential scaling of JOR for weak and strong items was unlikely.

Another possible explanation of factor $T$ is that subjects are sensitive to the degree to which a memory has consolidated and that they use this information to formulate a JOR (Wickelgren, 1972, 1974). The neural mechanisms 


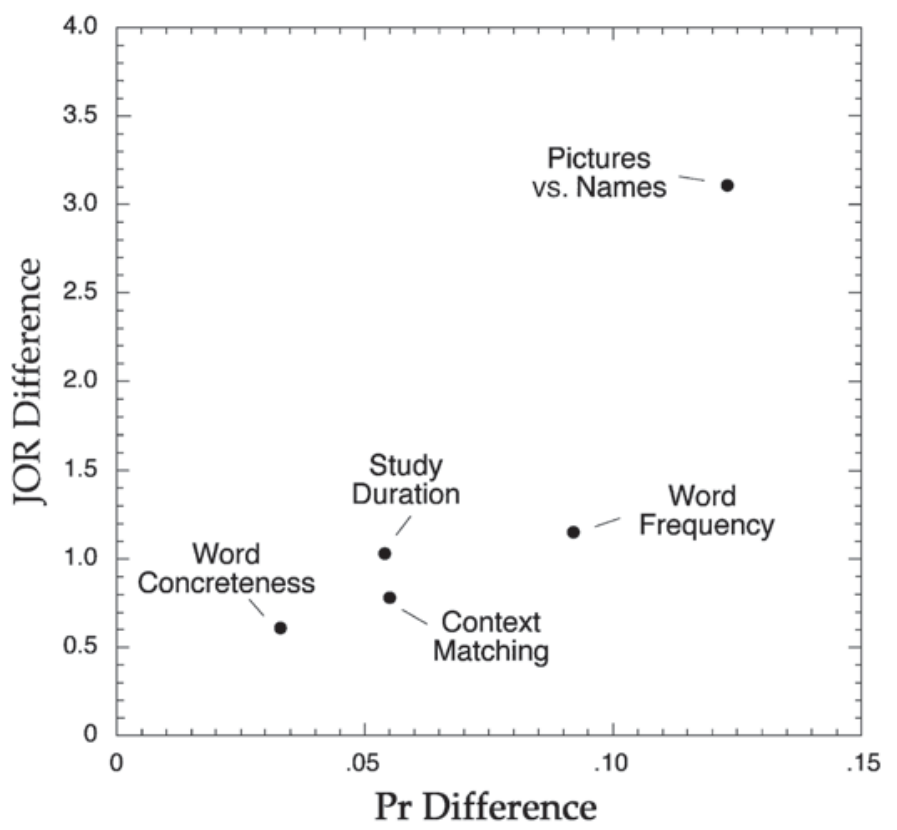

Figure 4. Scatter diagram of the difference in mean JOR versus the difference in mean recognition $\mathrm{Pr}$, across five experiments: word concreteness and word frequency from Hintzman (2003b); context matching from Hintzman (2002); pictures versus names and study duration data from the present experiments.

and anatomical systems that underlie a memory appear to change with time (e.g., McGaugh, 2000) and could therefore be seen as potential cues to recency. By itself, however, this hypothesis does not explain the activation pattern obtained in this and previous studies. The fact that weak items seem older than do strong items would require that they be more consolidated than strong items of the same age, and this seems like a paradox.

At the present, no one seems to have proposed a single mechanism for JOR that explains both its unique sensitivity to time and the highly replicable activation pattern. An alternative to the single-mechanism approach is to assume that apparent recency is an attribution that is based on more than one cue. In everyday life, as Friedman (1993) has argued, people use a variety of cues to judge when a remembered event occurred. These include associations with temporal locations and landmarks, relative order information, and information related directly to the memory's age, otherwise known as distance information.

Table 1

Percentage of Variance Among Means Explained by Linear Trend on Lag and by Item Manipulation

\begin{tabular}{cccc}
\hline Experiment & Dependent Variable & Lag & Item Manipulation \\
\hline Experiment 1 & JOR & 92 & 4 \\
& $P($ hit $)$ & 68 & 21 \\
Experiment 2 & JOR & 99 & 1 \\
& $P($ hit $)$ & 50 & 42 \\
\hline
\end{tabular}

The running judgment task used here is structured in a way that virtually excludes any role for information on location or order and thus emphasizes information related to distance.

The answer may be that, in making a JOR, subjects use two kinds of distance cues in parallel (Hintzman, 2004). Thus, Hartley's (1834) insight that a remembered idea's "vigour" is the cue to its age may be only part of the answer. The other hypothetical basis for JOR is the mysterious factor $T$. As hypothesized by G. D. A. Brown et al. (2000), factor $T$ could be based on oscillator readings, or, as hypothesized by Wickelgren $(1972,1974)$, it could be based on the degree of consolidation. Alternatively, factor $T$ could be a dynamically changing property of the memory trace that evolved specifically to represent a memory's age. Whatever the nature of factor $T$, the best current hypothesis seems to be that, in making a JOR, subjects allocate some weight to this time-related cue and some weight to strength or activation.

\section{REFERENCES}

Brown, G. D. A., Preece, T., \& Hulme, C. (2000). Oscillator-based memory for serial order. Psychological Review, 107, 127-181.

Brown, N. R., Rips, L. J., \& Shevell, S. K. (1985). The subjective dates of natural events in very-long-term memory. Cognitive Psychology, 17, 139-177.

FleXser, A. J., \& Bower, G. H. (1974). How frequency affects recency judgments: A model for recency discrimination. Journal of Experimental Psychology, 103, 706-716.

FozARD, J. L., \& WEINERT, J. R. (1972). Absolute judgments of recency 
for pictures and nouns after various numbers of intervening items. Journal of Experimental Psychology, 95, 472-474.

Friedman, W. J. (1993). Memory for the time of past events. Psychological Bulletin, 113, 44-66.

Glanzer, M., \& AdAMs, J. K. (1985). The mirror effect in recognition memory. Memory \& Cognition, 13, 8-20.

HaRTLEY, D. (1834). Observations on man, his fame, his duty, and his expectations (6th ed.). London: Thomas Tegg \& Son.

HinRICHS, J. V. (1970). A two-process memory-strength theory for judgment of recency. Psychological Review, 77, 223-233.

Hintzman, D. L. (2000). Memory judgments. In E. Tulving \& F. I. M. Craik (Eds.), The Oxford handbook of memory (pp. 165-177). Oxford: Oxford University Press.

Hintzman, D. L. (2002). Context matching and judgments of recency. Psychonomic Bulletin \& Review, 9, 368-374.

Hintzman, D. L. (2003a, November). Effects of repetition on judgment of recency. Paper presented at the annual meeting of the Psychonomic Society, Vancouver, British Columbia, Canada.

Hintzman, D. L. (2003b). Judgments of recency and their relation to recognition memory. Memory \& Cognition, 31, 26-34.
Hintzman, D. L. (2004). Time versus items in judgment of recency. Memory \& Cognition, 32, 1298-1304.

Loftus, G. R., \& Masson, M. E. J. (1994). Using confidence intervals in within-subject designs. Psychonomic Bulletin \& Review, 1, 476490.

McGaugh, J. L. (2000). Memory-A century of consolidation. Science, 287, 248-251.

Rosenthal, R., \& Rosnow, R. L. (1985). Contrast analysis: Focused comparisons in the analysis of variance. Cambridge: Cambridge University Press.

Schneider, B., Parker, S., Ostrosky, D., Stein, D., \& Kanow, G. (1974). A scale for the psychological magnitude of number. Perception \& Psychophysics, 16, 43-46.

WiCKELGREN, W. A. (1972). Trace resistance and the decay of long-term memory. Journal of Mathematical Psychology, 9, 418-455.

Wickelgren, W. A. (1974). Single-trace fragility theory of memory dynamics. Memory \& Cognition, 2, 775-780.

(Manuscript received September 29, 2004; revision accepted for publication January 15, 2005.) 